\title{
Préoccupations relayées par les associations de professeurs de langues vivantes outre-Manche (1880-1918)
}

\section{Adèle Thomas}

\section{(2) OpenEdition Journals}

Édition électronique

URL : https://journals.openedition.org/dhfles/1101

DOI : $10.4000 /$ dhfles. 1101

ISSN : 2221-4038

Éditeur

Société Internationale pour l'Histoire du Français Langue Étrangère ou Seconde

Édition imprimée

Date de publication : 1 décembre 2005

Pagination : 91-102

ISSN : 0992-7654

\section{Référence électronique}

Adèle Thomas, «Préoccupations relayées par les associations de professeurs de langues vivantes outre-Manche (1880-1918) ", Documents pour l'histoire du français langue étrangère ou seconde [En ligne], 35 | 2005, mis en ligne le 14 août 2011, consulté le 27 mai 2021. URL : http:// journals.openedition.org/dhfles/1101; DOI : https://doi.org/10.4000/dhfles.1101

Ce document a été généré automatiquement le 27 mai 2021.

(c) SIHFLES 


\title{
Préoccupations relayées par les associations de professeurs de langues vivantes outre-Manche (1880-1918)
}

\author{
Adèle Thomas
}

1 Au cours du XIX siècle, les enseignants qui exercent outre-Manche s'organisent et les professeurs de langues vivantes n'échappent pas à ce mouvement de fond qui donne naissance à de nombreuses sociétés professionnelles. Peu étudiées, ces associations ne sont tout de même pas totalement absentes des travaux consacrés à l'histoire de l'enseignement des langues en Angleterre. Deux d'entre elles ont ainsi été retenues au titre de leur contribution à la réforme et à l'amélioration de l'enseignement des langues: la Société Nationale des Professeurs de français en Angleterre (SNPF), créée par des Français en 1881; la Modern Language Association (MLA), qui voit le jour en 1892.

2 Mises en avant pour leur combat en faveur des méthodes dites "nouvelles " ${ }^{1}$, il ne faudrait pourtant pas en déduire que, lors des années qui précèdent la Première Guerre mondiale, ces deux associations œuvrent de concert, en seule quête de progrès méthodologique. L'étude des publications respectives de la MLA et de la SNPF suggère en effet que, si toutes deux semblent poursuivre un objectif globalement identique, leurs préoccupations ne convergent pas toujours et ne se limitent pas non plus à des questions de méthode.

3 Cet article fait suite à une communication au colloque de Valence dans laquelle mon propos concernait essentiellement la SNPF (cf.Thomas 2005). Je m'attacherai donc ici à présenter des éléments de comparaison entre la SNPF et la MLA. 


\section{Association contre groupe de pression?}

4 À première vue, la MLA et la SNPF se ressemblent. Créées par des enseignants soucieux d'extraire leur discipline d'un certain mépris (le français pour les membres de la SNPF ; les langues vivantes pour les membres de la MLA), les deux associations adoptent des moyens d'action identiques : congrès, réunions, publication d'un journal. Et, si la SNPF ne milite qu'en faveur de la langue française, les ambitions de la MLA et de la SNPF relèvent du même ordre: il s'agit d'améliorer l'enseignement des langues, de promouvoir leur apprentissage dans les écoles et d'obtenir pour elles comme pour les enseignants la place qui leur revient dans le système éducatif(Modern Quarterly of Language and Literature 1898, I, $2: 161$ ).

La composition de ces associations est cependant tout à fait différente. La SNPF ne veut ainsi compter parmi ses membres que des enseignants français, qu'elle recrute sur des critères de compétence et d'honorabilité. La MLA s'adresse quant à elle à tous ceux qu'intéresse la question des langues vivantes, quelles que soient leur nationalité et leur profession (ibid). Cette dernière rassemble donc plus de monde - y compris des Français de la SNPF(Modern Language Quarterly 1900, III, $3: 238-244$ ) - et passe ainsi de 390 à 900 membres entre 1901 et 1910(Modern Language Teaching 1906, II, 1: 7 ; MLT (1910, VI, 1 : 1), tandis qu'en 1906 la SNPF regroupe 310 personnes(Le Français 1906, $158: 2$ ).

6 Or ce choix des membres n'est pas sans conséquences : ouverte aux seuls Français, la SNPF s'expose rapidement à de sévères critiques. Nombreux sont ceux qui disqualifient en effet l'action d'une association qu'ils assimilent plutôt à un groupe de pression, dont les positions ne peuvent être motivées que par les intérêts des Sociétaires. Aujourd'hui encore, un chercheur tel qu'Eric Hawkins estime qu'en appelant au développement de l'oral dans l'apprentissage des langues, les Français ne font que prêcher pour leur paroisse. Il salue en revanche l'action de la MLA, créée par des anglophones dont il loue l'érudition, la capacité à se tourner vers l'avenir, ainsi que le dévouement aux nouvelles méthodes (Hawkins éd. 1987 : 121-122). Contrasté et donc fort intelligible, ce tableau n'en masque cependant pas moins bien des nuances.

\section{Une ferveur plus ou moins ardente pour l'innovation}

7 Malgré ses ambitions et malgré son nom (Modern Language Association), la MLA ne se défait jamais totalement de certaines traditions en matière éducative. Elle ne cesse ainsi de se préoccuper de la place du latin et du grec en cas de succès de la cause des langues vivantes. Il est vrai que l'association compte parmi ses membres de nombreux représentants d'une élite scolaire et universitaire, très attachée à la formation classique de la jeunesse anglaise. Au cours de l'assemblée générale de 1906, le président de l'association fait ainsi l'éloge des deux langues mortes et n'hésite pas à proclamer que ces dernières sont irremplaçables... L'une des chevilles ouvrières de la MLA s'empresse donc de déclarer qu'il faut en fait conclure de ces propos que les langues classiques et modernes gagneraient à être étudiées de manière conjointe (MLT 1906, II, $1:$ 9-10). Contrairement à la SNPF où l'on considère la chose acquise, la MLA s'interroge beaucoup sur la valeur éducative des langues vivantes : est-elle comparable à celle du latin et du grec? Elle se donne ainsi pour mission de prouver sans relâche à l'opinion publique que les langues vivantes n'ont pas qu'une valeur utilitaire (MLT 1905, I, 1 : 27-28). 
8 De telles résistances au changement se manifestent également dans les discours qui traitent des méthodes d'apprentissage qu'il conviendrait d'adopter. La MLA se prononce assez vite en faveur de l'ajout de tests oraux dans les examens de langues vivantes. Toutefois, de nombreux membres expriment leurs craintes quant au développement de l'oral. En 1899, l'un d'entre eux met ainsi en doute la qualité intellectuelle d'un tel enseignement et s'inquiète : il estime en effet qu'un imbécile qui apprend à parler le français et l'allemand, loin de progresser sur la voie de la sagesse, ne gagne en fait rien d'autre que le pouvoir d'exprimer sa bêtise en trois langues(MQLL 1899, I, $4: 314)$. La critique n'est pas isolée. Les nouvelles méthodes ne font vraiment pas l'unanimité parmi ceux qui pensent que seuls six mois passés à l'étranger permettent de faire de réels progrès à l'oral ou bien encore ceux qui souhaitent que l'enseignement de la littérature soit privilégié(MLQ 1900, III, 3 : 169 ; MQLL 1898, I, 1 : 63). L'association présente pourtant les différentes méthodes à ses membres, étourdis parfois par ce qu'ils nomment en français un « embarras de richesse » (MLQ 1901, IV, 2 : 109), mais ceux-ci ne semblent pas être en mesure de parvenir à un accord. À la suite d'un échange de lettres acides, l'un d'entre eux rappelle d'ailleurs que la MLA a finalement résolu d'adopter une position de neutralité en la matière (MLT 1911, VII, 6 : 183-184).

9 Tous ces débats méthodologiques n'affectent pas l'unité de la SNPF, dont les membres pensent tous peu ou prou - et comme leurs prédécesseurs semble-t-il (Radford 1985 : 205-206) - qu'il faut surtout tendre à un équilibre. La SNPF prône en effet un enseignement « vivant » des langues qui, sans exclure la grammaire, la littérature ou la traduction, devrait également s'enrichir d'une partie de civilisation.

10 En fait, les Sociétaires se méfient des méthodes (Hamonet 1882:3) et ils mettent donc en garde leurs confrères contre tout abus de la sorte. Dans un article intitulé «Les Professeurs peints par leurs livres", l'un d'entre eux se moque ainsi des soi-disant innovateurs qui découvrent enfin les vertus de l'oral. Il dénonce par ailleurs les excès d'un auteur (confrère anglais de la MLA) qui, pour expliquer le mot 'utile' sans le traduire en anglais, «tournera autour du pot avec plus d'ambages et de circonlocutions encore que le professeur de philosophie de M. Jourdain »(Le Français 1903, $136: 12$ ).

11 L'intervention des membres de la SNPF dans le journal de la MLA montre d'ailleurs bien la modération que préconise l'association des Français : l'on y trouve ainsi des articles sur l'abus du livre et de l'anglais dans l'enseignement du français en Angleterre, sur la nécessité d'aborder dans ces cours des éléments de géographie, d'histoire et de littérature(MQLL 1898, I, $3: 235-237$ et 1899, I, $4: 334-336$; MLT 1905, I, 2 et $3: 43-52)$. De même, à une époque où la MLA redoute toute dérive de type utilitaire, la SNPF ne craint pas d'investir tous les champs de la langue. Ainsi la création du Grand Concours de Langue et de Littérature françaises n'empêche pas l'association d'établir un examen commercial en 1895 (Le Français 1899, 120 : 19).

12 Au début du XXe siècle, la SNPF consacre un article à ce qu'elle appelle «La Nouvelle Méthode ». C'est l'occasion de rappeler qu'il n'y a là rien de nouveau pour l'association, qui s'efforce de faire savoir depuis 20 ans qu'il convient « d'enseigner une langue parlée en la parlant » (ibid. 1903, 139 : 1). Pour autant, les Sociétaires sont invités à ne pas se faire spectateurs des luttes pédagogiques en cours.

13 En somme, si les associations œuvrent dans le sens d'une certaine réforme, leur soutien aux innovations méthodologiques s'avère parfois bien réservé. Et d'ailleurs, la question n'est pas seule à mobiliser toute leur attention. 


\section{Luttes d'influence sur le terrain linguistique}

14

Contrairement à la SNPF qui ne milite que pour la cause du français, la MLA a pour ambition de défendre plusieurs langues et s'interroge. Dans quel ordre convient-il d'apprendre ces langues? L'organe de la MLA recèle de nombreuses suggestions: commencer par le français et poursuivre avec l'allemand puis le latin; enseigner le français en une partie du pays et l'allemand dans l'autre, puis passer au latin et au grec; débuter l'allemand à 10 ans, ajouter le latin à 12 ans et choisir le grec ou le français à 14 ans, etc. (MLT 1911, VII, 1: 14 ; MQLL 1899, I, $4: 301$; MLT 1905, I, 2 et $3: 57$ ). Presque toutes les combinaisons possibles sont présentées.

Mais la question qui, dans ce domaine, mobilise le plus d'énergie au sein de la MLA est indéniablement celle du déclin de l'allemand en Angleterre(MLQ 1897, I, 1: 8-13; MQLL 1899, I, $4: 328 ;$ MLT 1907, III, $8: 254)$. Si certains accusent l'Entente cordiale de ne pas y être étrangère(MLT 1906, II, $7: 209$ ), l'association alerte en fait ses lecteurs sur le sort de l'allemand depuis plusieurs années déjà, alors même que la SNPF conseille aux professeurs de français en mal de poste d'étudier l'allemand afin d'ajouter une corde à leur arc (voir infra). La MLA, qui se défend de tout parti pris pour l'allemand au détriment du français (MLT 1908, IV, $3: 69,75$ ) et qui se distingue de la SNPF en ce qu'elle ne se présente pas comme un regroupement national, n'est pourtant pas exempte de toute influence. Fondée sous la présidence du professeur d'origine allemande Max Müller (The Year Book of Modern Languages 1920 : 192), elle doit beaucoup à l'activité d'anciens étudiants ayant suivi l'enseignement de Karl Breul et du Dr. Braunholtz à Cambridge (MLT 1909, V, $6: 174$ ). Par ailleurs en 1900, environ un tiers des membres de la MLA appartient également à une société allemande qui souhaite épurer la langue allemande des mots étrangers et répandre l'amour de l'Allemagne dans le monde (MLQ 1900, III, $3: 186$ ).

Le choix de l'allemand comme première langue vivante (alors que traditionnellement cette place revient au français) fait donc l'objet de nombreux articles dans l'organe de la MLA. La langue et la culture allemandes n'y sont jamais présentées au travers de leur altérité. Il s'agit au contraire de les inscrire dans une certaine proximité avec la culture anglaise, en des temps où l'on estime que l'école doit être un lieu d'acquisition de l'identité nationale. L'apprentissage de l'allemand est ainsi recommandé au nom d'une parenté ethnique et linguistique.

Présenté comme une langue proche de l'anglais, l'allemand serait non seulement plus facile que le français (MLT 1905, I, 2 et $3: 55-56$ ), mais aussi d'un intérêt plus grand pour les Anglais en raison d'une communauté de race : «the English and the Germans are really one race " (ibid. 1907, III, $5: 147$ ).La langue et la culture allemandes sont ainsi valorisées en qualité d'éléments originels, constitutifs du patrimoine anglais. Chaque Anglais qui est en mesure de le faire est même appelé à se rendre au moins une fois dans sa vie au pays de ses ancêtres, afin de se rendre compte des formidables qualités du peuple allemand (MQLL 1899, I, 4 : 328). L'apprentissage de l'allemand comme première langue vivante est annoncé comme une réalité qui, dans le prolongement d'un mouvement amorcé outre-Atlantique, ne saurait tarder(MLQ 1902, V, 1 : 39).

Tous ces arguments ne parviennent cependant pas à remporter l'adhésion du Board of Education puisqu'en 1909, le ministère se prononce en faveur d'un maintien du statu quo (MLT 1909, V, 4 : 105). Si la MLA s'avère donc ici peu influente, l'association marque 
cependant des points dans un autre débat qui, depuis longtemps, agite le milieu des enseignants de langues vivantes.

\section{Qui doit enseigner les langues vivantes?}

19 En 1907, la MLA convie deux membres de la SNPF à siéger dans une commission qui étudie la question de la préparation des professeurs à l'enseignement des langues vivantes (Le Français 1908, $165: 7$; MLT 1909, V, 3 : 66). La chose mérite d'être soulignée car il s'agit justement du point sur lequel les deux associations se divisent tout particulièrement.

Tout en affirmant qu'il ne suffit pas d'être français pour enseigner sa langue (Le Français 1903, 139 : 6), la SNPF se prononce fermement en faveur du recrutement majoritaire de Français, estimant que seuls ces derniers sont à même de bien enseigner la langue et la culture françaises. Cette condition n'est pas spécifiquement dirigée contre les professeurs de nationalité anglaise : ceux-ci s'avèrent en effet bien peu nombreux à l'époque de formation de la SNPF. L'exigence de nationalité française s'inscrit plutôt en réaction à la perception d'une certaine intrusion des exilés du continent sur le marché de l'enseignement du français en Angleterre (SNPF, Congrès, 1885: 41). Et le ressentiment des Sociétaires s'avère d'autant plus fort lorsque ces professeurs de français viennent d'outre-Rhin. Marqué par la défaite de 1870, l'un d'eux affirme ainsi que «les Allemands et leurs congénères offensent la conscience publique lorsqu'ils affichent l'audacieuse prétention d'enseigner la langue française » (Hamonet 1882:5).

21 Si la MLA s'ouvre à tous, sans restriction nationale, elle n'en évalue pas moins elle aussi la situation à partir de ce critère de nationalité : les étrangers ne conviennent pas car ils font l'objet de moqueries s'ils ne parlent pas bien l'anglais ; les Anglais ne sauraient cependant les remplacer, faute de posséder les compétences requises. Aussi est-il d'abord envisagé de mettre en place une coopération: les Anglais se chargeraient de maintenir la discipline dans les petites classes, puis ils passeraient la main à leurs homologues étrangers dans les classes plus avancées (MQLL 1898, I, 1: 62).

Mais le souci d'inscrire l'enseignement des langues vivantes dans un cadre national inspire rapidement de nouvelles orientations. L'opinion se répand que, quel que soit le niveau d'apprentissage, un Anglais compétent s'avère toujours préférable ${ }^{2}$. Les enseignants immigrés sont tenus responsables du sort peu enviable des langues vivantes (MLT 1905, I, 2 et $3: 68$ ), et l'on conclut que cet enseignement gagnerait en dignité s'il était exercé par des professeurs ayant effectué leur cursus universitaire en Angleterre (MQLL 1899, I, 4 : 309).

Il ne s'agit pourtant pas d'évacuer totalement les étrangers du système scolaire : leur présence apparaît en effet indispensable à l'apprentissage d'une bonne prononciation. Prenant exemple sur le système du maître allemand secondé par un Lektor étranger ${ }^{3}$, il est ainsi conseillé de toujours s'adjoindre les services d'un jeune étranger de bonne éducation(MLQ 1903, VI, 1 : 38). La SNPF envisage elle aussi une coopération sous l'angle de la subordination, mais elle s'appuie sur les principes de la Méthode directe pour justifier la mise en place d'un rapport inversé... faisant du maître anglais l'aide du maître étranger (Le Français 1903, 139 : 6).

Les assemblées générales de la MLA sont parfois l'occasion de discours virulents à l'égard des professeurs étrangers. Aux lecteurs qui font alors part de leur indignation dans le journal de l'association, celle-ci répond que si l'un des présidents de la MLA 
s'est exprimé en faveur des Anglais sans la moindre retenue, il ne faut cependant pas en déduire que l'association partage ces vues. Celle-ci se défend en effet de vouloir écarter les enseignants du continent et affirme ne pas avoir d'avis sur la question : son seul souci serait d'exclure le professeur incompétent, quelle que soit sa nationalité(MQLL 1899, II, $5: 66$ ). Et pourtant les attaques se poursuivent ; les Sociétaires ne manquent pas de s'en plaindre ${ }^{4}$.

Conscients des nouvelles exigences que suscite l'évolution de leur activité, ces derniers s'adaptent. La SNPF, qui se charge aussi de placer ses membres, explique ainsi aux Français en recherche de poste que la seule maîtrise du français ne suffit pas :

La moitié au moins des demandes qui nous arrivent requièrent une bonne connaissance de la langue allemande. Nous ne saurions donc trop engager les futurs candidats à étudier consciencieusement cette langue.(Le Français 1905, $148: 12)^{5}$

Pareil conseil indique clairement que l'association s'éloigne là de ses premiers préceptes. Il devient en effet difficile d'exiger qu'une langue vivante ne soit enseignée que par celles et ceux dont elle est langue première dès lors que l'on invite les Français à enseigner au moins deux langues vivantes. Ainsi, au fil des ans, la SNPF revoit ses positions, atténue sa rigueur et s'ouvre aux autres nationalités. En 1906, elle institue un Certificat d'aptitude à l'enseignement de la langue française en Angleterre, expliquant que " puisque l'enseignement du français ne peut être confié, dans toutes les écoles, à des professeurs ou à des institutrices de nationalité française, ceux du moins qui auront obtenu notre diplôme de capacité posséderont toutes les connaissances et toute l'expérience désirables pour nous aider dans notre tâche, éliminer les incapables, et faire enfin de cet enseignement ce que l'on en attend: un levier d'éducation pratique, intellectuel et moral. » Toutefois la SNPF ne se cache pas que, par ce biais, elle entend bien sauvegarder son prestige et demeurer l'arbitre "en tout ce qui touche à l'enseignement du français en Angleterre.» (ibid. 1906, $156: 10$ ). À partir de 1912, il n'est désormais plus obligatoire d'être français pour appartenir à la SNPF.

L'association ne cesse pourtant pas de vouloir défendre l'intérêt des Français. À l'occasion de discussions sur la mise en place d'une liste de professeurs habilités à enseigner en Angleterre, certains Sociétaires font ainsi valoir qu'il serait bon que la SNPF soit représentée par un député au Teachers' Registration Council afin de ne pas voir les Français noyés dans «la masse indigène " (ibid., 1914, 199: 6-7). Il est vrai que l'absence de représentation ne réussit pas aux native teachers. En raison de la guerre, ces derniers sont peu consultés au cours de l'enquête qui conduit à la rédaction du rapport sur l'enseignement des langues vivantes en Grande-Bretagne (The Year Book of Modern Languages $1920: 2$, Leathes Report) ... et la teneur de ce rapport décisif s'en ressent. Les absents sont ainsi accusés de privilégier, pour l'enseignement des langues vivantes, une approche traditionnelle et archaïque. On leur reproche de ne pas savoir maintenir la discipline dans leurs classes, mais aussi de ne pas délivrer un enseignement conforme aux besoins, aux traditions éducatives et au tempérament de la nation. La conclusion de ce rapport est sans appel : il faut remplacer les étrangers par des Britanniques (Leathes $1918:$ 43-44).

La MLA se réjouit ouvertement de cette décision et ne manque pas de reproduire les paragraphes du rapport qui détaillent tous les défauts des native teachers dans le Year Book of Modern Languages qu'elle publie en 1920 (The Year Book of Modern Languages 1920 : 6-7). Au lendemain de la Première Guerre mondiale, il n'est désormais plus question 
pour l'association de se réfugier derrière la neutralité dont elle se réclamait encore à l'aube du XXe siècle.

À une époque où les méthodes d'enseignement des langues suscitent bien des débats outre-Manche, il est tentant d'imaginer que des associations d'enseignants telles que la MLA et la SNPF se mobilisent entièrement autour de ce thème et qu'elles prennent clairement position. Soucieuses d'œuvrer en faveur de la reconnaissance de leur activité et de leur discipline, les deux associations ne se limitent pourtant pas à l'étude de cette question, que l'une a bien du mal à trancher et que l'autre semble avoir dépassé. La formation et la sélection des enseignants, le choix des langues à enseigner font ainsi l'objet de moult analyses et propositions. Les divergences d'opinion qui apparaissent alors entre l'association des Français et celle des anglophones nous invitent à nous interroger quant à leurs motivations profondes. Tandis que la MLA, créée dans un esprit d'ouverture manifeste, se présente comme une association impartiale, la SNPF fait figure de groupe de pression, guidé par le seul intérêt de ses membres. Pourtant, la MLA s'avère ne pas être exempte de toute influence et, sous couvert de défendre toutes les langues vivantes, elle n'en milite pas moins pour l'une d'entre elles essentiellement. Par ailleurs, tandis que la SNPF s'ouvre progressivement à l'idée que l'enseignement du français puisse ne pas être réservé aux seuls Français, la MLA n'hésite plus, au lendemain de la guerre, à se prononcer en faveur d'une éviction des native teachers, au nom de l'inscription de l'enseignement des langues vivantes dans un cadre national. Qu'elles s'en réclament ou s'en défendent, ces deux associations se révèlent fort marquées par les questions d'identité nationale, d'influence culturelle... et leurs considérations pédagogiques s'en ressentent.

\section{BIBLIOGRAPHIE}

\section{Sources primaires}

Le Français (bulletin de la SNPF).

Journal of Education.

Modern Language Quarterly, Modern Quarterly of Language and Literature, Modern Language Teaching (organes de la MLA).

HAMONET, Alfred. 1882. Réflexions sur l'enseignement du français en Angleterre, texte du discours prononcé à Londres le 13 janvier 1882 à l'occasion du Congrès des professeurs de langue française (12 au 14/01/1882). Londres.

LEATHES, Stanley Mordaunt - Great Britain Board of Education - Committee on the Position of Modern Languages in the Educational System of Great Britain. 1918. Report of the Committee appointed by the Prime Minister to enquire into the Position of Modern Languages in the Educational System of Great Britain, Londres : His Majesty's Stationery Office.

SNPF, Congrès de la Société Nationale des Professeurs de français en Angleterre - Quatrième session, tenue à Londres les 2 et 3 janvier 1885. Londres: Hachette. 
SNPF, Statuts et règlement, votés en AG à Londres le 12/01/1882.

The Year Book of Modern Languages (1920),edited for the Council of the MLA by G. Waterhouse. Cambridge : Cambridge UP.

\section{Sources secondaires}

ARMYTAGE, W.H.G. 1970 (1 $1^{\text {ère }}$ éd. 1964). Four Hundred Years of English Education, Cambridge : CUP. BAYLEY, Susan. 1998. « The Direct Method and Modern Language Teaching in England 1880-1918». History of Education, 27, $1: 39-57$.

BERGEN, Barry H. 1982. « Only a Schoolmaster : Gender, Class, and the Effort to Professionalize Elementary Teaching in England, 1870-1910 ». History of Education Quarterly, 22, 1: 1-21 BRUNOT, Ferdinand. 1905-. Histoire de la langue française, t. V, VIII et XI, Paris : A. Colin. GERBOD, Paul. 1988. « L’Enseignement de la langue française en Grande-Bretagne au XIX ${ }^{\mathrm{e}}$ siècle (1800-1870)», Documents $2: 8-11$. GERBOD, Paul. 1991. «L'Enseignement de la langue française en Angleterre au XIX siècle (1800-1860) ». L'Information historique 53, 5 : 180-186.

HAWKINS, Eric. 1987 (1 1 ère éd. 1981. Modern Languages in the Curriculum, Cambridge : Cambridge UP. LAMBLEY, Kathleen. 1920. The Teaching and Cultivation of the French Language in England during Tudor and Stuart Times, with an introductory chapter on the preceding period, Manchester : UP.

LAWSON, John, Silver, Harold. 1973. A Social History of Education in England, Londres : Methuen.

RADFORD, H. 1985. « Modern Languages and the Curriculum in English Secondary Schools ». In GOODSON, I. (dir.). Social Histories of the Secondary Curriculum, Londres : Falmer Press, 203-237. THOMAS, Adèle. 2005. «À la conquête d'un statut professionnel : les enseignants de français en Angleterre et leurs associations (1880-1914)». Documents 33-34 : 214-226.

\section{NOTES}

1. Bayley (1998 : 45-46) ; Hawkins éd. (1987 : 121-122) ; Radford (1985 : 213, 217-218).

2. MQLL 1898, I, $2: 144$; MQLL 1899, I, $4: 302$, 308 ; MLQ 1901, IV, $2: 105$ et 1902, V, $1: 42:$ « Our education must be a national education; only an Englishman can be inspired by the genuine and enlightened love of his country which must pervade our teaching; only he will be able to make English boys and girls feel how much there is to admire in the people and the literature of France and Germany. »

3. Système présenté au cours de l'assemblée générale de la MLA en 1899.

4. Le Français 1903, 139: 12: "On ne voit qu'une chose en Angleterre: il faut remplacer les Français par les Anglais. C'est la mort de la connaissance pratique de la langue française dans ce pays-ci. »

5. Les recommandations de ce type sont nombreuses. Voir aussi : ibid. 1904, $144: 8$ et 1907, 164 : 8. 


\section{RÉSUMÉS}

Deux associations bien distinctes, constituées à l'initiative de professeurs de langues vivantes, voient le jour en Angleterre à la fin du XIX ${ }^{\mathrm{e}}$ siècle : la Société Nationale des Professeurs de français en Angleterre, créée par des Français, et la Modern Language Association. Quelles sont leurs préoccupations en des temps où l'on discute beaucoup de méthodes d'apprentissage des langues? L'étude des journaux de ces deux sociétés dévoile le champ de leurs considérations pédagogiques, inscrites sur fond de luttes d'influence culturelle.

Two distinct societies, initiated by modern foreign language teachers, appear in England at the end of the $19^{\text {th }}$ century: the Société Nationale des Professeurs de français en Angleterre, created by French exiles, and the Modern Language Association. What are the issues raised among those circles in times when learning methods are very much discussed for modern languages? The study of the journals published by those associations unveils a whole breadth of reflections, in line with a general struggle for cultural influence.

\section{INDEX}

Mots-clés : Professeurs de langues vivantes, Angleterre, associations, influence culturelle Keywords : Foreign language teachers, England, associations, cultural influence

\section{AUTEUR}

\section{ADÈLE THOMAS}

Université Paris XIII 